\title{
VIRIAL IDENTITIES FOR NONLINEAR SCHRÖDINGER EQUATIONS WITH A CRITICAL COEFFICIENT INVERSE-SQUARE POTENTIAL
}

\author{
TOSHIYUKI SUZUKI
}

Abstract. Virial identities for nonlinear Schrödinger equations with some strongly singular potential $\left(a|x|^{-2}\right)$ are established. Here if $a=a(N):=-(N-2)^{2} / 4$, then $P_{a(N)}:=-\Delta+a(N)|x|^{-2}$ is nonnegative selfadjoint in the sense of Friedrichs extension. But the energy class $D((1+$ $\left.\left.P_{a(N)}\right)^{1 / 2}\right)$ does not coincide with $H^{1}\left(\mathbb{R}^{N}\right)$. Thus justification of the virial identities has a lot of difficulties. The identities can be applicable for showing blow-up in finite time and for proving the existence of scattering states.

Mathematics subject classification (2010): 35Q55, 35Q40, 81Q15.

Keywords and phrases: virial identities, nonlinear Schrödinger equations, Hartree equations, inversesquare potentials, blow-up in finite time, scattering states, Mellin transform.

\section{REFERENCES}

[1] N. Burq, F. Planchon, J. Stalker, A. S. TAhVildar-Zadeh, Strichartz estimates for the wave and Schrödinger equations with the inverse-square potential, J. Funct. Anal. 203 (2003), 519-549.

[2] T. Cazenave, Semilinear Schrödinger Equations, Courant Lecture Notes in Mathematics, 10. New York University, Courant Institute of Mathematical Sciences, New York; American Mathematical Society, 2003.

[3] T. Cazenave, F. B. Weissler, Rapidly decaying solutions of the nonlinear Schrödinger equation, Comm. Math. Phys. 147 (1992), 75-100.

[4] J. Ginibre, G. Velo, On a class of nonlinear Schrödinger equations with nonlocal interaction, Math. Z. 170 (1980), 109-136.

[5] R. T. GLASSEY, On the blowing up of solutions to the Cauchy problem for nonlinear Schrödinger equations, J. Math.P̉hys. 18 (1977), 1794-1797.

[6] N. Hayashi, T. OzAWA, Scattering theory in the weighted $L^{2}\left(\mathbb{R}^{n}\right)$ spaces for some Schrödinger equations, Ann. Inst. Henri Poincaré 48 (1988), 17-37.

[7] N. Hayashi, Y. Tsutsumi, Scattering theory for Hartree type equations, Ann. Inst. Henri Poincaré 46 (1987), 187-213.

[8] E. H. Lieb, B. Simon, The Hartree-Fock theory for Coulomb systems, Comm. Math. Phys. 53 (1977), 185-194.

[9] N. OKaZawa, T. Suzuki, T. Yокота, Cauchy problem for nonlinear Schrödinger equations with inverse-square potentials, Appl. Anal. 91 (2012), 1605-1629.

[10] N. OKAZAWA, T. SuZUKI, T. YокоTA, Energy methods for abstract nonlinear Schrödinger equations, Evol. Equ. Control Theory, 1 (2012), 337-354.

[11] F. Planchon, J. Stalker, A. S. TAhVIlddar-ZAdeh, $L^{p}$ estimates for the wave equation with the inverse-square potential, Discrete Continuous Dyn. Systems 9 (2003), 427-442.

[12] E. M. Stein, G. Weiss, "Introduction to Fourier Analysis on Euclidean Spaces," Princeton University Press, Princeton, NJ, 1971.

[13] T. SUZUKI, Energy methods for Hartree type equation with inverse-square potentials, Evol. Equ. Control Theory, 2 (2013), 531-542.

[14] T. SuZuKI, Blowup of nonlinear Schrödinger equations with inverse-square potentials, Differ. Equ. Appl., 6 (2014), 309-333. 
[15] T. SUZUKI, Solvability of nonlinear Schrödinger equations with some critical singular potential via generalized Hardy-Rellich inequalities, Funkcial. Ekvac., 59 (2016), 1-34.

[16] T. SuzUKI, Scattering theory for Hartree equations with inverse-square potentials, Appl. Anal., 96 (2017), 2032-2043.

[17] J. ZhANG, J. Zheng, Scattering theory for nonlinear Schrödinger equations with inverse-square potential, J. Funct. Anal. 267 (2014), 2907-2932. 